\title{
A GALOIS-DYNAMICS CORRESPONDENCE FOR UNICRITICAL POLYNOMIALS
}

\author{
ROBIN ZHANG
}

\begin{abstract}
In an analogy with the Galois homothety property for torsion points of abelian varieties that was used in the proof of the Mordell-Lang conjecture, we describe a correspondence between the action of a Galois group and the dynamical action of a rational map. For nonlinear polynomials with rational coefficients, the irreducibility of the associated dynatomic polynomial serves as a convenient criterion, although we also verify that the correspondence occurs in several cases when the dynatomic polynomial is reducible. The work of Morton, Morton-Patel, and Vivaldi-Hatjispyros in the early 1990s connected the irreducibility and Galois-theoretic properties of dynatomic polynomials to rational periodic points; from the Galois-dynamics correspondence, we derive similar consequences for quadratic periodic points of unicritical polynomials. This is sufficient to deduce the non-existence of quadratic periodic points of quadratic polynomials with exact period 5 and 6 , outside of a specified finite set from Morton and Krumm's work in explicit Hilbert irreducibility.
\end{abstract}

\section{INTRODUCTION}

1.1. A general definition. In arithmetic geometry, it is natural to consider the action of Galois groups on torsion points of abelian varieties. The proof of the Mordell-Lang conjecture crucially uses the fact that torsion points of abelian varieties always satisfy the following Galois homothety property (cf. Lan65, Ser00, Théorème 2], [Hin88, Lemme 12], McQ95, Theorem 2.1.6], [Poo99, Section 1]): for an abelian variety $A$ defined over a number field $k$, there exists an $i \geq 1$ such that for all positive integers $m$, there is a $\sigma_{m} \in \operatorname{Gal}(\bar{k} / k)$ such that

$$
\sigma_{m}(x)=m^{i} x
$$

for all $\bar{k}$-rational points $x$ on $A$ of finite order coprime to $m$.

For arithmetic dynamical systems, we introduce an analogous correspondence with two goals in mind. First, we establish a "dynamical Galois homothety property", designated the Galois-dynamics correspondence, as a weaker and slightly more prevalent condition than the irreducibility of dynatomic polynomials studied in the work of Vivaldi-Hatjispyros VH92. Second, we explore the connections between the Galois-dynamics correspondence and quadratic periodic points.

Let $\phi: \mathbf{P}^{1} \rightarrow \mathbf{P}^{1}$ be a rational map over a field $k$ and let $\phi^{N}$ denote the $N$-th iterate of $\phi$, so $\phi^{N}:=\phi \circ \phi^{N-1}$. For $S$ a subset of an algebraic closure $\bar{k}$ of $k$, let $\operatorname{Per}_{N, S}(\phi)$ denote the periodic points in $S$ of $\phi$ of exact period $N$.

Definition 1.1. Let $\phi: \mathbf{P}^{1} \rightarrow \mathbf{P}^{1}$ be a rational map over a field $k, N$ be an integer greater than 1 , and $K / k$ be a nontrivial Galois extension. For a periodic point $z \in$ $\operatorname{Per}_{N, K-k}(\phi)$, the tuple $(\phi, N, K / k, z)$ satisfies the Galois-dynamics correspondence

Date: May 18, 2021. 
(GDC) if and only if there is a positive integer $i<N$ and a nontrivial $\sigma \in \mathrm{Gal}(K / k)$ such that

$$
\sigma(z)=\phi^{i}(z)
$$

Furthermore, we say that the triple $(\phi, N, K / k)$ satisfies the Galois-dynamics correspondence if and only if $(\phi, N, K / k, z)$ satisfies the Galois-dynamics correspondence for every $z \in \operatorname{Per}_{N, K-k}(\phi)$.

Unlike the Galois homothety property, which always holds in the setting of abelian varieties, the Galois-dynamics correspondence is not always satisfied for dynamical systems. Even if we restrict our attention to unicritical polynomials, which can be written as $\phi_{d, c}(z):=z^{d}+c$, there are instances in which the Galoisdynamics correspondence might not be satisfied.

Example 1.2. If $K$ is the splitting field of $\phi_{2, t}^{3}(z)-z$ over the rational function field $k=\mathbf{C}(t)$, then the directed graph of periodic points of $\phi_{2, t}$ of exact period $N=3$ consists of two disjoint 3-cycles. A result of Bousch Bou92, Chapter 3, Theorem 3] implies that the Galois group $\operatorname{Gal}(K / \mathbf{C}(t))$ properly contains the full automorphism group of this graph and, in particular, contains an element $\sigma$ of order 2 that interchanges the two 3-cycles. If $k$ is the fixed field $K^{\sigma}$, then $[K: k]=2$. For any $z \in \operatorname{Per}_{N, K-k}(\phi)$ of exact period 3 , the $\operatorname{Gal}(K / k)$-orbit of $z$ is $\{z, \sigma(z)\}$, but $\sigma(z)$ is not in the forward orbit of $z$ with respect to $\phi_{2, t}$. Hence $\left(\phi_{2, t}, 3, K / K^{\sigma}\right)$ does not satisfy the Galois-dynamics correspondence.

Remark 1.3. The definition of the Galois-dynamics correspondence can be naturally generalized by allowing $\phi$ to be any endomorphism on an algebraic variety. If $A$ is an abelian variety and $[m]$ denotes its multiplication-by- $m$ endomorphism, then the Galois homothety property due to Faltings and Serre (cf. McQ95, Theorem 2.1.6]) implies that for all integers $m$ and $N$ greater than $1,([m], N, \bar{k} / k)$ satisfies the Galois-dynamics correspondence.

Remark 1.4. If $\phi$ is a polynomial with coefficients in $k$, then its iterative action commutes with the action of $\operatorname{Gal}(K / k)$. In particular, $(\phi, N, K / k, z)$ satisfies the Galois-dynamics correspondence if and only if the $N$-cycle of $z$ is not disjoint from its conjugates by some element of the Galois group, i.e.

$$
\left\{z, \ldots, \phi^{N-1}(z)\right\} \cap\left\{\sigma(z), \ldots, \sigma\left(\phi^{N-1}(z)\right)\right\} \neq \emptyset,
$$

for some nontrivial $\sigma \in \operatorname{Gal}(K / k)$.

1.2. Irreducibility and the main theorem. We will focus on the classical case when $k=\mathbf{Q}$ and $\phi$ is a nonlinear polynomial in $\mathbf{Q}[z]$. First, we recall the definition of dynatomic polynomials. An $N$-periodic point $z$ of $\phi$ is always a root of the polynomial $\phi^{n}(z)-z$ for all multiples $n$ of $N$. By the Möbius inversion formula, we have a factorization of $\phi^{n}(z)-z$ in terms of the $N$-th dynatomic polynomial $\Phi_{N}(z)$

$$
\begin{aligned}
\phi^{n}(z)-z & =\prod_{N \mid n} \Phi_{N}(z) \in \mathbf{Q}[z] \\
\Phi_{N}(z): & =\prod_{m \mid N}\left(\phi^{m}(z)-z\right)^{\mu(N / m)} \in \mathbf{Q}[z],
\end{aligned}
$$

where $\mu$ is the Möbius function. The roots of $\Phi_{N}(z)$ are called the periodic points of $\phi$ of formal period $N$, which generically are the periodic points of $\phi$ of exact 
period $N$. When we study the family of unicritical polynomials, with $\phi=\phi_{d, t}$, we can consider the dynatomic polynomial $\Phi_{N}(z, t)$ as an element of $\mathbf{Q}[z, t]$. The zero locus of $\Phi_{N}(z)$ defines an affine curve with a smooth projective model denoted $C_{1}(N)$ that carries an action of $\mathbf{Z} / N \mathbf{Z}$ induced by the iteration of $\phi$. We can then define the quotient $C_{0}(N)$ of $C_{1}(N)$ by this action.

The Galois-dynamics correspondence holds in the case considered by VivaldiHatjispyros [VH92, namely when $\phi$ is any nonlinear polynomial in $\mathbf{Q}[z]$ and its associated dynatomic polynomial $\Phi_{N}(z)$ is irreducible in $\mathbf{Q}[z]$. In Section 2.1, we slightly modify a result of Vivaldi-Hatjispyros to obtain the following irreducibility criterion for the Galois-dynamics correspondence.

Proposition 1.5 (Proposition 2.2). Let $\phi$ be any nonlinear polynomial and let $N$ be an integer greater than 1 . If the dynatomic polynomial $\Phi_{N}(z)$ is irreducible in $\mathbf{Q}[z]$ then $(\phi, N, K / \mathbf{Q})$ satisfies the Galois-dynamics correspondence for all nontrivial Galois extensions K/Q.

Therefore, the Galois-dynamics correspondence can be viewed as a weaker notion than the irreducibility of $\Phi_{N}(z)$. Irreducibility is sufficient to provide most of the cases of the Galois-dynamics correspondence, since it is often the "typical" situation. For $\phi$ belonging to certain families of polynomials in $\mathbf{Q}[z]$, including the unicritical polynomials $\phi_{d, c}$, the dynatomic polynomial $\Phi_{N}(z, t)$ is known to be irreducible in $\mathbf{C}(t)[z]$ by the work of Bousch [Bou92, Chapitre 3, Théorème 1], Lau-Schleicher [LS94, Theorem 4.1], and Morton [Mor96, Theorem B]. So for these families of polynomials, we apply Hilbert's irreducibility theorem in Section 2.2 to show that $\left(\phi_{d, c}, N, K / \mathbf{Q}\right)$ satisfies the Galois-dynamics correspondence for all Galois extensions $K / \mathbf{Q}$ so long as $c$ lies outside of a subset of $\mathbf{Q}$ of asymptotic density 0 , called the exceptional set $\Sigma_{d, N}$.

The main theorem of this paper establishes cases of the Galois-dynamics correspondence for quadratic polynomials with various $N$, even when $\Phi_{N}$ is reducible and $c$ is in the exceptional set $\Sigma_{2, N}$. Its proof in Section 4 combines the irreducibility criterion of Proposition 1.5 with descriptions of the exceptional sets $\Sigma_{2, N}$ due to Morton Mor92 and Krumm Kru18, Kru19] to establish the Galois-dynamics correspondence when $c \in \mathbf{Q}-\Sigma_{2, N}$. For the exceptional cases $c \in \Sigma_{2, N}$, an explicit understanding of how $\Phi_{N}(z, c)$ factors for fixed specializations $t=c$ is used to check the Galois-dynamics correspondence directly on a case-by-case basis.

Theorem 1.6. For the quadratic polynomial $\phi_{2, c}(z)=z^{2}+c$ with rational coefficients and all nontrivial Galois extensions $K / \mathbf{Q},\left(\phi_{2, c}, N, K / \mathbf{Q}\right)$ satisfies the Galois-dynamics correspondence in the following cases:

- $N=2:$ all $c \in \mathbf{Q}$;

- $N=3$ : all $c \in \mathbf{Q}$;

- $N=4$ :

$$
\begin{aligned}
& -[K: \mathbf{Q}]=2: \text { all } c \in \mathbf{Q} ; \\
& -[K: \mathbf{Q}]>2: \text { all } c \notin\left\{\frac{-s^{3}-2 s+4}{4 s} \mid s \in \mathbf{Q}^{\times}\right\} ;
\end{aligned}
$$

- $N=5,6,7$, or 9 : all but finitely many $c \in \mathbf{Q}$.

1.3. Quadratic points and periodic points. Theorem 1.6 has a nice consequence in the dynamics of quadratic polynomials over quadratic number fields. For periods 5 and 6 , no periodic points of $\phi_{2, c}$ are believed to exist in quadratic number fields besides the single known 6 -cycle, as suggested by extensive numerical evidence 
by Doyle-Faber-Krumm [DFK14, Hutz-Ingram [HI13, and Wang-Zhang WZ15, Section 5], in addition to the theoretical results of Doyle Doy18, Doy19, Doy20 and Krumm Kru16.

Conjecture 1.7. Let $K$ be a quadratic number field.

(a) If $c \in \mathbf{Q}$, then $\phi_{2, c}$ has no periodic points of exact period 5 in $K$.

(b) If $c \in \mathbf{Q}-\left\{-\frac{71}{48}\right\}$, then $\phi_{2, c}$ has no periodic points of exact period 6 in $K$.

Let $C_{1}(N)$ denote the dynatomic modular curve parametrizing pairs $\left(\phi_{d, c}, z_{0}\right)$ of unicritical polynomials and periodic points of period $N$, and let $C_{0}(N)$ denote the dynatomic modular curve parametrizing pairs $\left(\phi_{d, c}, \mathcal{O}\right)$ of unicritical polynomials and $N$-cycles. There is a pair of projections

$$
C_{1}(N) \longrightarrow C_{0}(N) \longrightarrow \mathbf{P}^{1} .
$$

In Section 3, we show that if $K$ is a quadratic number field and $\left(\phi_{d, c}, N, K\right)$ satisfies the Galois-dynamics correspondence, then the fiber above $c \in \mathbf{P}^{1}(\mathbf{Q})$ on $C_{1}(N)(K)$ maps to a point in $C_{0}(N)(\mathbf{Q})$. Therefore, if the points of $C_{0}(N)(\mathbf{Q})$ are completely known for a fixed $N$ and $\left(\phi_{d, c}, N, K\right)$ is known to satisfy the Galois-dynamics correspondence for all $c \in \mathbf{Q}$, then one would be able to completely determine the periodic points of $\phi_{d, c}$ in $K$ of period $N$. In this fashion, the Galois-dynamics correspondence acts as a bridge between the $\mathbf{Q}$-rational points of $C_{0}(N)$ and the $K$-rational points of $C_{1}(N)$. Since the points of $C_{0}(N)(\mathbf{Q})$ are well-understood for for $N=5$ and $N=6$ due to Flynn-Poonen-Schaefer [FPS97] and Stoll [Sto08, the possible counterexamples to Conjecture 1.7 are constrained by the finite sets $\Sigma_{2,5}$ and $\Sigma_{2,6}$.

Corollary 1.8. Let $K$ be a quadratic number field. If $c \in \mathbf{Q}-\Sigma_{2,5}$, then $\phi_{2, c}$ has no periodic points of exact period 5 in $K$.

Corollary 1.9. Let $K$ be a quadratic number field and let $J$ be the Jacobian of $C_{0}(6)$. Assume that the $L$-series $L(J, s)$ extends to an entire function, $L(J, s)$ satisfies the standard functional equation, and the weak Birch and Swinnerton-Dyer conjecture is valid for $J$. If $c \in \mathbf{Q}-\left(\Sigma_{2,6} \cup\left\{-\frac{71}{48}\right\}\right)$, then $\phi_{2, c}$ has no periodic points of exact period 6 in $K$.

Remark 1.10. A priori, the statement of Corollary 1.8 can be recovered from the finiteness of the exceptional set $\Sigma_{2,5}$ from Krumm [Kru19], since the 30 periodic points of exact period 5 must then be conjugate (and therefore not quadratic) for all but finitely many rational $c$. For Corollary 1.9, Doyle-Faber-Krumm [DFK14, Section 1.4] observed that the results of Stoll Sto08 actually imply that there are only finitely many pairs $(K, c)$ of quadratic number fields $K$ and $c \in K$ such that $\phi_{2, c}$ has a periodic point of exact order 6 in $K$.

1.4. Future directions. If the Galois-dynamics correspondence can be established for all $c \in \mathbf{Q}$ for given pairs $(d, N)$, then future developments in determining the structure of $C_{0}(N)(\mathbf{Q})$ and $C_{1}(N)(\mathbf{Q})$ could lead to more general statements of Poonen's conjecture (cf. Poo98) for number fields. It is not known if there are any tuples $\left(\phi_{2, c}, N, K / \mathbf{Q}\right)$ that do not satisfy the Galois-dynamics correspondence, but we show that $c$ must lie in the density 0 subset $\Sigma_{2, N}$ of the rationals for any such tuple. There are two parallel directions that would directly extend Theorem 1.6 and provide more cases of the Galois-dynamics correspondence: further 
identifying the exceptional sets $\Sigma_{d, N}$ and direct verification of the Galois-dynamics correspondence for the exceptional cases $c \in \Sigma_{d, N}$.

In the first direction (for $d=2$ ), the program of Krumm Kru18, Kru19 in extending the results of Morton Mor92 has yielded significant progress in determining the exceptional sets $\Sigma_{2, N}$ for small $N$. The finiteness methods developed by Krumm are constrained by computational limitations for larger $N$ (cf. Kru19, Section 9]). However, further calculation of the exceptional sets $\Sigma_{2, N}$ ("explicit Hilbert irreducibility") would yield immediate extensions of Theorem 1.6 for $N>4$. In particular, Corollaries 1.8 and 1.9 would be immediately refined by the explicit determination of $\Sigma_{2,5}$ and $\Sigma_{2,6}$.

In the second direction, the techniques in Section 4 for checking the exceptional cases of $c \in \Sigma_{2, N}$ when $N \leq 4$ have been ad-hoc and depend on explicit factorizations of $\Phi_{N}(z, c)$ in $\mathbf{Q}[z]$. Any case-by-case approach for the exception cases may depend on progress in the first direction, since an explicit description for the exceptional set $\Sigma_{d, N}$ is not known if $N>4$ or $d>2$. However, it is worth pointing out that if $\left(\phi_{2, c}, 5, K / \mathbf{Q}\right)$ and $\left(\phi_{2, c}, 6, K / \mathbf{Q}\right)$ satisfy the Galois-dynamics correspondence for all $c \in \Sigma_{2,5}$ and $\Sigma_{2,6}$ respectively, then Conjecture 1.7 would be affirmatively resolved (assuming the standard conjectures for $N=6$ ).

Outside of those two directions, the Galois-dynamics correspondence can also be considered for broader classes of functions $\phi$. For instance, the results of Section 2.2 for unicritical polynomials $\phi_{d, c}$ and their consequences would also extend to any nonlinear polynomial $\phi(z)$ whose associated dynatomic polynomial $\Phi_{N}(z, t)$ is known to be irreducible over $\mathbf{Q}(t)$. Furthermore, Definition 1.1 of the Galoisdynamics correspondence is made in sufficiently generality that this program can also be considered for dynamical systems over finite fields, dynamical systems over function fields, and more general dynamical systems on algebraic varieties. There are, for instance, interesting calculations done by by Bridy-Garton [BG17] and Krumm-Sutherland KS21] that could be used for such analogues.

\section{IRREDUCIBILITY}

2.1. An irreducibility criterion. The dynamical mimicry of the action of the Galois group of $\Phi_{N}$ was previously considered by Vivaldi-Hatjispyros for any nonlinear polynomial $\phi$. They established that if the dynatomic polynomial $\Phi_{N}(z)$ is irreducible as a polynomial in $\mathbf{Q}[z]$ for a given $N$, then for each orbit $\mathcal{O}$ there is a nontrivial subgroup $H_{\mathcal{O}}$ of the Galois group of $\Phi_{N}(z)$ that acts on $\mathcal{O}$ in the same way as iterates of $\phi$ VH92, Section 3]. As a consequence of the work of VivaldiHatjispyros and the following general fact, there is an irreducibility criterion for the Galois-dynamics correspondence.

Lemma 2.1. Let $\phi$ be a polynomial in $k[z], N$ be an integer greater than 1 , and $K^{\prime}$ be the splitting field of the dynatomic polynomial $\Phi_{N}$ over $k$. If $\left(\phi, N, K^{\prime} / k\right)$ satisfies the Galois-dynamics correspondence, then $(\phi, N, K / k)$ satisfies the Galoisdynamics correspondence for all nontrivial Galois extensions $K / k$.

Proof. First, we show that $(\phi, N, L / k)$ satisfies the Galois-dynamics correspondence for any nontrivial intermediate Galois extension $L$ of $K^{\prime} / k$. If $\operatorname{Per}_{N, L-k}(\phi)$ is empty, then $(\phi, N, L / k)$ vacuously satisfies the Galois-dynamics correspondence, so we may assume that $\operatorname{Per}_{N, L-k}(\phi)$ is nonempty. Let $z_{0} \in \operatorname{Per}_{N, L-k}(\phi) \subset \operatorname{Per}_{N, K^{\prime}-k}(\phi)$. By the definition of the Galois-dynamics correspondence for $\left(\phi, N, K^{\prime} / k, z_{0}\right)$, there 
is a positive integer $i<N$ and a nontrivial $\tau^{\prime} \in \operatorname{Gal}\left(K^{\prime} / k\right)$ such that $\phi^{i}\left(z_{0}\right)=$ $\tau^{\prime}\left(z_{0}\right)$. Notice that $\tau^{\prime}$ cannot be in the subgroup $\operatorname{Gal}\left(K^{\prime} / L\right)$, since $\tau^{\prime}$ does not fix $z_{0}$, which is an element of $K$. Then $\tau^{\prime}$ corresponds to a nontrivial element $\tau$ of the quotient group $\operatorname{Gal}(L / k)$ such that $\phi^{i}\left(z_{0}\right)=\tau\left(z_{0}\right)$. Therefore $(\phi, N, L / k)$ satisfies the Galois-dynamics correspondence for any nontrivial intermediate Galois extension $L / k$ of $K^{\prime} / k$.

Now consider the arbitrary nontrivial Galois extension $K / k$, for which we can similarly assume that $\operatorname{Per}_{N, K-k}(\phi)$ is nonempty. Let $z_{0} \in \operatorname{Per}_{N, K-k}(\phi)$. Since $z_{0}$ is a root of $\Phi_{N}(z)$, it is also contained in $\operatorname{Per}_{N, K^{\prime}-k}(\phi)$. For the intermediate Galois extension $\left(K \cap K^{\prime}\right) / k$, the triple $\left(\phi, N,\left(K \cap K^{\prime}\right) / k\right)$ satisfies the Galois-dynamics correspondence and $z_{0} \in \operatorname{Per}_{N,\left(K \cap K^{\prime}\right)-k}(\phi)$. Then there is a positive integer $i<N$ and a nontrivial $\sigma^{\prime} \in \operatorname{Gal}\left(\left(K \cap K^{\prime}\right) / k\right)$ such that $\phi^{i}\left(z_{0}\right)=\sigma^{\prime}\left(z_{0}\right)$. By taking a lift $\sigma \in \operatorname{Gal}(K / k)$ of $\sigma^{\prime},(\phi, N, K / k)$ satisfies the Galois-dynamics correspondence.

Proposition 2.2 (Irreducibility criterion). Let $\phi$ be any nonlinear polynomial in $\mathbf{Q}[z], N$ be an integer greater than 1 , and $K / \mathbf{Q}$ be a nontrivial Galois number field. If the dynatomic polynomial $\Phi_{N}(z)$ is irreducible in $\mathbf{Q}[z]$, then $(\phi, N, K / \mathbf{Q})$ satisfies the Galois-dynamics correspondence.

Proof. Let $K^{\prime}$ be the splitting field of $\Phi_{N}$ over Q. Vivaldi-Hatjispyros VH92, Section 3] proved that if $\Phi_{N}(z)$ is irreducible in $\mathbf{Q}[z]$, then for each orbit $\mathcal{O}$ there is a nontrivial subgroup $H_{\mathcal{O}}$ of the Galois group of $\Phi_{N}(z)$ that acts on $\mathcal{O}$ in the same way as iterates of $\phi$. Consider any periodic point $z_{0} \in \operatorname{Per}_{N, K^{\prime}-\mathbf{Q}}(\phi)$ and its orbit $\mathcal{O}$. Then there is a nontrivial $\sigma \in H_{\mathcal{O}} \subset \operatorname{Gal}\left(K^{\prime} / \mathbf{Q}\right)$ such that $\sigma(z)=\phi^{i}(z)$ for some positive integer $i<N$, so $\left(\phi, N, K^{\prime} / \mathbf{Q}\right)$ satisfies the Galois-dynamics correspondence. By Lemma 2.1, $(\phi, N, K / \mathbf{Q})$ satisfies the Galois-dynamics correspondence as well.

Remark 2.3. The notion of an automorphism polynomial, which was defined by the contemporaneous study of Morton-Patel MP94 for non-constant polynomials $\phi \in k[z]$, is also closely related to the Galois-dynamics correspondence when $\Phi_{N}(z)$ is irreducible in $k[z]$. If $\Phi_{N}(z)$ is irreducible in $k[z]$ and $\phi$ is an automorphism polynomial of $\Phi_{N}(z)$, then for $K^{\prime}$ the splitting field of $\Phi_{N}$ over $k,\left(\phi, N, K^{\prime} / k\right)$ satisfies the Galois-dynamics correspondence with $i=1$ for each periodic point $z_{0}$ of exact period $N$. By Lemma 2.1, this is actually sufficient for $(\phi, N, K / k)$ to satisfy the Galois-dynamics correspondence for any nontrivial Galois extension $K / k$.

2.2. Hilbert irreducibility and the exceptional set for unicritical polynomials. For unicritical polynomials $\phi_{d, c}$, Vivaldi-Hatjispyros [VH92, Section 3] remarked that the dynatomic polynomial $\Phi_{N}(z, c)$ being irreducible in $\mathbf{Q}[z]$ "appears to be the typical situation", meaning that it is reducible for $c$ in a density 0 subset of $\mathbf{Q}$. They proved their hypothesis for $d=2$ and $N \leq 3$, but nevertheless $\Phi_{N}(z, c)$ is not irreducible in $\mathbf{Q}[z]$ in general. Even for $d=2$, Vivaldi-Hatjispyros demonstrated that $\Phi_{N}(z, c)$ is never irreducible in $\mathbf{Q}[z]$ for an infinite family of $c$ when $N=3$, for $c=-2$ when $N \geq 3$, and for $c=0$ when $2^{N}-1$ is not a Mersenne prime.

To study the irreducibility of $\Phi_{N}(z, c) \in \mathbf{Q}[z]$ for $c \in \mathbf{Q}$, we can view it as a specialization of $\Phi_{N}(z, t) \in \mathbf{Q}[z, t]$. By the later results of Bousch Bou92, Chapitre 3, Théorème 1] and Lau-Schleicher [LS94, Theorem 4.1] (cf. Morton Mor96, Corollary 1], Buff-Lei [BL14, Theorem 1.2], Gao-Ou GO14, Theorem 1.2]), it is known 
that $\Phi_{N}(z, t)$ is irreducible as a polynomial in $\mathbf{C}(t)[z]$. Let $G_{N}$ denote the Galois group of $\Phi_{N}(z, t)$ over $\mathbf{Q}(t)$ and let $G_{N, c}$ denote the Galois group of its specialization $\Phi_{N}(z, c)$ over $\mathbf{Q}$. Define $\Sigma_{d, N}$ to be the locus of $c \in \mathbf{P}^{1}(\mathbf{Q})$ such that $G_{N} \cong G_{N, c}$. The thinness (in the sense of Serre) of $\Sigma_{d, N}$ follows immediately from an application of Hilbert's irreducibility theorem.

Corollary 2.4. For each integer $d$ and $N$ greater than $1, \Sigma_{d, N}$ is a thin subset (in the sense of Serre) of $\mathbf{P}^{1}(\mathbf{Q})$ such that for all rational $c$ not in $\Sigma_{d, N}$,

(a) $\Phi_{N}(z, c)$ is irreducible in $\mathbf{Q}[z]$, and

(b) $G_{N} \cong G_{N, c}$.

Remark 2.5. Thin sets (in the sense of Serre) in $\mathbf{P}^{1}(\mathbf{Q})$ have density 0 , as the number of points of a thin set in $\mathbf{P}^{1}(\mathbf{Q})$ with naive height at most $H$ is $\mathrm{O}(H)$ (cf. Serre [Ser08, Proposition 3.4.2]). Corollary 2.4 implies that, for all $N$, the set of rational $c$ such that $\Phi_{N}(z, c)$ is reducible in $\mathbf{Q}[z]$ is a density 0 subset of $\mathbf{Q}$.

When $\Phi_{N}(z, c)$ is irreducible in $\mathbf{Q}[z]$, the Galois group $G_{N, c}$ acts transitively on the periodic points of $\phi_{d, c}$ of period $N$. We obtain the following useful statement as a consequence of Proposition 2.2 and Corollary 2.4.

Proposition 2.6. For each integer $d$ and $N$ greater than 1 , there exists a thin set (in the sense of Serre) $\Sigma_{d, N}$ in $\mathbf{P}^{1}(\mathbf{Q})$ such that for all rational c not in $\Sigma_{d, N}$,

(a) the triple $\left(\phi_{d, c}, N, K / \mathbf{Q}\right)$ satisfies the Galois-dynamics correspondence for all nontrivial Galois extensions $K / \mathbf{Q}$, and

(b) $G_{N} \cong G_{N, c}$.

Remark 2.7. In fact, Corollary 2.4 (and therefore Proposition 2.6) is true for a larger class of polynomials than the unicritical polynomials $\phi_{d, c}$. By Morton Mor96. Theorem B] (cf. Morton [Mor98b, Theorem B]), the associated dynatomic polynomial $\Phi_{N}(z, t)$ is also irreducible in $\mathbf{C}(t)[z]$ for polynomials $\phi \in \mathbf{Z}[z, t]$ of degree $d \geq 2$ satisfying:

(a) $\phi\left(z, t^{i}\right)$ is homogeneous for some integer $i \geq 1$,

(b) $\phi(z, 0)=z^{d}$,

(c) $\phi(z, 1)$ has distinct roots, and

(d) the primitive $N$-bifurcation points of $\phi$ are distinct.

This class of polynomials includes $\phi_{d, c}=z^{d}+c$ (by setting $c=-t^{d}$ ).

Understanding when $G_{N} \not G_{N, c}$ determines the exceptional set $\Sigma_{d, N}$. On the one hand, the structure of the Galois group $G_{N}$ of $\Phi_{N}(z, t)$ over $\mathbf{Q}(t)$ is wellunderstood. Bousch [Bou92, Chapitre 3] (c.f. [MP94, Theorem 4.2]) showed that the Galois group $G_{N}$ of $\Phi_{N}(z, t)$ over $\mathbf{Q}(t)$ is isomorphic to a wreath product

$$
G_{N} \cong(\mathbf{Z} / N \mathbf{Z}) \curlywedge S_{r},
$$

where $r$ is an integer such that $r N=\operatorname{deg} \Phi_{N}$, for $\phi_{2, c}$. This result was extended by Lau-Schleicher [LS94] (cf. Morton [Mor98b]) for $\phi_{d, c}$ to all $d \geq 2$.

However, the structure of the Galois group $G_{N, c}$ of the specialization $\Phi_{N}(z, c)$ over $\mathbf{Q}$ is not known in general. For $d=2$, one can show that $G_{N, c}$ is not isomorphic to $G_{N}$ for any integer $N>1$ if $c=0$ or -2 (i.e. that $0,-2 \in \Sigma_{2, N}$ for all $N>1$ ) since the Galois group $G_{N, c}$ is abelian for those values of $c$. Otherwise, there are only explicit descriptions of $\Sigma_{2, N}$ for small $N$ due to Morton [Mor92 and Krumm Kru18, Kru19. 


\section{QUADRATIC POINTS ON DYNATOMIC CURVES}

The study of periodic points of exact period $N$ in a number field $K$ is related to the study of $K$-points on the curves $C_{1}(N)$ and $C_{0}(N)$ : a pair $\left(z_{0}, \phi_{d, c}\right)$ of a map $\phi_{d, c}(z)=z^{d}+c$ with $c \in K$ and a periodic point $z_{0} \in K$ of exact period $N$ of $\phi_{d, c}$ gives rise to a $K$-point on $C_{1}(N)$, and a pair $\left(\mathcal{O}, \phi_{d, c}\right)$ of a map $\phi_{d, c}(z)=z^{d}+c$ with $c \in K$ and a $\operatorname{Gal}(\bar{K} / K)$-stable $N$-cycle $\mathcal{O}$ gives rise to a $K$-point on $C_{0}(N)$. The $K$-points on these curves contain full information about periodic points in $K$.

The first observation about the Galois-dynamics correspondence is that we can specify the dynamical indices corresponding to the Galois action. The following elementary lemma is true in greater generality for any rational map $\phi$ and any nontrivial finite Galois extension $K / k$, but we state it in the unicritical polynomial and number field setting.

Lemma 3.1. Let $K$ be a nontrivial finite Galois extension of $\mathbf{Q}$ of degree $D$, let $d$ and $N$ be integers greater than 1 , let $c \in \mathbf{Q}$, and denote $g:=\operatorname{gcd}(N, D)$. Let $z_{0} \in K-\mathbf{Q}$ be a periodic point of $\phi_{d, c}$ with exact period $N$.

If there exists a nontrivial $\sigma \in \operatorname{Gal}(K / \mathbf{Q})$ and a positive integer $i<N$ such that

$$
\sigma z_{0}=\phi_{d, c}^{i}\left(z_{0}\right)
$$

then $g>1$ and $i=\frac{m N}{g}$ for some integer $m$ such that $1 \leq m \leq g-1$.

Proof. Suppose that there exists a nontrivial $\sigma \in \operatorname{Gal}(K / \mathbf{Q})$ such that $\phi_{d, c}^{i}\left(z_{0}\right)=$ $\sigma\left(z_{0}\right)$ for some $i \in\{1, \ldots, N-1\}$. Since $\sigma$ commutes with $\phi_{d, c}$ and the action of $\phi_{d, c}$ is transitive on the $N$-cycle, we have that $\sigma \equiv \phi_{d, c}^{i}$ on the entire cycle.

Since $K$ is Galois, the order of the Galois group $\operatorname{Gal}(K / \mathbf{Q})$ is $D$. Thus,

$$
z_{0}=\sigma^{D}\left(z_{0}\right)=\left(\phi_{d, c}^{i}\right)^{D}\left(z_{0}\right)=\phi_{d, c}^{i D}\left(z_{0}\right) .
$$

Since $z_{0}$ has exact period $N$, it follows that $N$ divides $i D$ and $i$ is a multiple of $\frac{N}{g}$. In particular, there is a contradiction if $g=1$ since $0<i<N$ by assumption.

Remark 3.2. In other words, $\phi_{d, c}$ satisfies the Galois-dynamics correspondence for $N, K$, and $z_{0} \in K-\mathbf{Q}$ if and only if there exists a nontrivial $\sigma \in \operatorname{Gal}(K / \mathbf{Q})$ and $m \in\{1, \ldots, g-1\}$ such that $\phi_{d, c}^{\frac{m N}{g}}\left(z_{0}\right)=\sigma z_{0}$ (this fact becomes convenient for showing Lemma 4.5). In particular, a necessary condition for $\left(\phi_{d, c}, N, K / \mathbf{Q}, z_{0}\right)$ to satisfy the Galois-dynamics correspondence is that $N$ and $[K: \mathbf{Q}]$ are not coprime.

If $K$ is a quadratic number field, then we can use Lemma 3.1 to make the following observation.

Lemma 3.3. Let $K$ be a quadratic number field, $c$ be a rational number, and $d$ and $N$ be integers greater than 1 . If $\left(\phi_{d, c}, N, K / \mathbf{Q}\right)$ satisfies the Galois-dynamics correspondence, then:

(a) For any $N$-cycle $\left\{z_{0}, \ldots z_{N-1}\right\}$ of $\phi_{d, c}$ in $K-\mathbf{Q}$, its trace $\sum z_{i}$ is rational;

(b) Furthermore, each $N$-periodic point $z_{i}$ is rational if $N$ is odd.

Proof. (a): If $N$ is even, the $N$-cycle consists of pairs of Galois conjugates $\left(z_{i}, z_{i+\frac{N}{2}}\right)$ by Lemma 3.1 Then the trace $\sum_{i=0}^{N-1} z_{i}$ must lie in $\mathbf{Q}$.

(b): If $N$ is odd, then the set of periodic points $\operatorname{Per}_{N, K-\mathbf{Q}}\left(\phi_{d, c}\right)$ must be empty because otherwise, $i \in\{1, \ldots, N-1\}$ would be a multiple of $N$ by virtue of the 
fact that $g=\operatorname{gcd}(N, 2)=1$. Hence any periodic point of $\phi_{d, c}$ of odd exact period $N$ contained in $K$ must itself be rational.

Remark 3.4. Effectively, Lemma 3.3 is the observation that when $K$ is a quadratic extension, the Galois-dynamics correspondence says that the nontrivial automorphism of $K$ setwise fixes $N$-cycles in $K$. This forces the trace to be rational and induces an involution on each $N$-cycle in $K$, which must have a fixed point when $N$ is odd.

Remark 3.5. Lemma 3.3(a) can be restated as saying that if $c \in \mathbf{Q}$ and $\left(\phi_{d, c}, N, K\right)$ satisfies the Galois-dynamics correspondence, then the fiber above $c$ on $C_{1}(N)(K)$ maps to a point in $C_{0}(N)(\mathbf{Q})$. Lemma 3.3(b) can be restated as saying that if furthermore $N$ is odd, then the fiber above $c$ on $C_{1}(N)(K)$ is actually contained in $C_{1}(N)(\mathbf{Q})$.

Combined with existing results on the rational points of dynatomic curves, Lemma 3.3 immediately yields the connection between the Galois-dynamics correspondence and Conjecture 1.7 .

Corollary 3.6. Let $K$ be a quadratic number field and $c$ be a rational number. If $\left(\phi_{2, c}, 5, K\right)$ satisfies the Galois-dynamics correspondence, then $\phi_{2, c}$ has no periodic points of exact period 5 in $K$.

Proof. The parameter $c$ and the trace of an $N$-cycle give coordinates on $C_{0}(N)$. Since we assume that $c$ is a rational number, it is sufficient to show that the trace of an $N$-cycle is rational to demonstrate that an $N$-cycle corresponds to a point in $C_{0}(N)(\mathbf{Q})$.

By Lemma 3.3, a 5-cycle of $\phi_{2, c}$ necessarily has rational trace and corresponds to a $\mathbf{Q}$-point on $C_{0}(5)$. By the work of Flynn-Poonen-Schaefer [FPS97, the genus 2 dynatomic curve $C_{0}(5)$ has only six rational points, three of which correspond to $c=\infty$ and three of which ultimately correspond to 5 -cycles in quintic cyclic extensions of $\mathbf{Q}$.

Corollary 3.7. Let $J$ be the Jacobian of $C_{0}(6)$. Suppose that the $L$-series $L(J, s)$ extends to an entire function, $L(J, s)$ satisfies the standard functional equation, and the weak Birch and Swinnerton-Dyer conjecture is valid for $J$.

Let $K$ be a quadratic number field and $c$ be a rational number. If $\left(\phi_{2, c}, 6, K\right)$ satisfies the Galois-dynamics correspondence, then $\phi_{2, c}$ has no periodic points of exact period 6 in $K$, unless $K=\mathbf{Q}(\sqrt{33})$ and $c=-\frac{71}{48}$ in which case there is exactly one 6-cycle:

$$
z_{0}=-1+\frac{\sqrt{33}}{12}, z_{1}=-\frac{1}{4}-\frac{\sqrt{33}}{6}, z_{2}=-\frac{1}{2}+\frac{\sqrt{33}}{12}, z_{i+3}=\sigma\left(z_{i}\right)
$$

where $\sigma$ is the nontrivial element of $\operatorname{Gal}(K / \mathbf{Q})$.

Proof. By Lemma 3.3, the trace of a 6 -cycle of $\phi_{2, c}$ in a quadratic number field $K$ is rational so it would necessarily correspond to a point in $C_{0}(6)(\mathbf{Q})$. By the work of Stoll [Sto08, which is dependent on the usual conjectures for $L(J, s)$, there are only ten points in $C_{0}(6)(\mathbf{Q})$. Five of the points correspond to cusps on $C_{1}(6)$ and the other five points correspond to explicitly-described 6-cycles; the following cycle with $c=-\frac{71}{48}$ and $K=\mathbf{Q}(\sqrt{33})$ is the only one defined over a quadratic field:

$$
z_{0}=-1+\frac{\sqrt{33}}{12}, z_{1}=-\frac{1}{4}-\frac{\sqrt{33}}{6}, z_{2}=-\frac{1}{2}+\frac{\sqrt{33}}{12}, z_{i+3}=\sigma\left(z_{i}\right)
$$


where $\sigma$ is the nontrivial element of $\operatorname{Gal}(K / \mathbf{Q})$.

Remark 3.8. In the situations of Corollaries 3.6 and 3.7 the irreducibility criterion of Proposition 1.5 is not sufficient. For example, $\Phi_{6}(z, c)$ is reducible as a polynomial in $\mathbf{Q}[z]$ when $c=-2$.

Remark 3.9. Corollaries 1.8 and 1.9 follow immediately from the application of Theorem 1.6 to Corollaries 3.6 and 3.7

\section{The Proof of Theorem 1.6}

In Theorem 1.6, we want to show that the quadratic polynomial $\left(\phi_{2, c}, N, K / \mathbf{Q}\right)$ satisfies the Galois-dynamics correspondence for all nontrivial Galois extensions $K / \mathbf{Q}$ with various $N \leq 9$ and $c \in \mathbf{Q}$. For the proof, we proceed case-by-case for varying $N$. For each $N$, we first use Proposition 2.6 and descriptions of $\Sigma_{2, N}$ to show that $\left(\phi_{2, c}, N, K / \mathbf{Q}\right)$ satisfies the Galois-dynamics correspondence when $c \in \mathbf{Q}-\Sigma_{2, N}$, before then directly checking the $c \in \Sigma_{2, N}$.

\subsection{Period 2.}

Lemma 4.1. The tuple $\left(\phi_{2, c}, 2, K / \mathbf{Q}\right)$ satisfies the Galois-dynamics correspondence for all nontrivial Galois number fields $K / \mathbf{Q}$ and all $c \in \mathbf{Q}$.

Proof. For $N=2$, there can only be at most one 2-cycle of $\phi_{2, c}$ for a given $c \in$ $\mathbf{Q}$ since $\Phi_{2}(z, c)$ is a degree 2 polynomial in $\mathbf{Q}[z]$. Therefore either $\Phi_{2}(z, c)$ is irreducible or the periodic points are rational.

\subsection{Period 3.}

Lemma 4.2. The tuple $\left(\phi_{2, c}, 3, K / \mathbf{Q}\right)$ satisfies the Galois-dynamics correspondence for all nontrivial Galois number fields $K / \mathbf{Q}$ and all $c \in \mathbf{Q}$.

Proof. The dynatomic polynomial $\Phi_{3}(z, c) \in \mathbf{Q}[z]$ for a given $c \in \mathbf{Q}$ can either be irreducible or factor into two (possibly reducible) cubic factors (cf. VivaldiHatjispyros [VH92, Section 2] and Morton [Mor92, Theorem 3]). By Proposition 1.5. $\left(\phi_{2, c}, 3, K / \mathbf{Q}\right)$ satisfies the Galois-dynamics correspondence for any Galois number field $K / \mathbf{Q}$ if $\Phi_{3}(z, c)$ is irreducible in $\mathbf{Q}[z]$, so we can assume that $\Phi_{3}(z, c)$ factors into two cubic factors.

Any linear factor of $\Phi_{3}(z, c)$ corresponds to a periodic point in $\mathbf{Q}$. A 3-cycle of $\phi_{2, c}$ containing a rational number must necessarily be entirely contained in $\mathbf{Q}$, so $\phi_{2, c}$ has a 3-cycle entirely contained in $\mathbf{Q}$. We can disregard such a 3-cycle, since the Galois-dynamics correspondence only concerns irrational periodic points.

For any irreducible cubic factor of $\Phi_{3}(z, c)$, two of its roots must lie in the same 3 -cycle. Then $\phi_{2, c}$ satisfies the Galois-dynamics correspondence for that 3-cycle and any Galois number field $K / \mathbf{Q}$ containing it since the 3 -cycle is not disjoint from its $\sigma$-conjugates for some nontrivial $\sigma \in \operatorname{Gal}(K / \mathbf{Q})$. Alternatively, observe that if $\phi_{2, c}(z)=\sigma(z)$ then

$$
\phi_{2, c}^{2}(z)=\phi_{2, c}(\sigma(z))=\sigma \phi_{2, c}(z)=\sigma^{2}(z) .
$$

Remark 4.3. Walde-Russo [WR94, Corollary 2] and Vivaldi-Hatjispyros VH92, Section 5] showed that the Galois group of any irreducible cubic factor of $\Phi_{3}(z, c)$ is isomorphic to $A_{3}$. 
Remark 4.4. Morton [Mor92, Theorem 8] showed that

$\Sigma_{2,3}=\left\{0,-\frac{7}{2}\right\} \cup\left\{-\frac{r^{3}+29 r^{2}+243 r+559}{16(r+7)(r+11)} \mid r \in \mathbf{Q}-\{-7,-11\}\right\} \cup\left\{-\frac{s^{2}+7}{4} \mid s \in \mathbf{Q}\right\}$.

In fact, Morton showed that while $G_{N, c}$ is not isomorphic to the full wreath product when $c=0,-\frac{7}{2}$, or $-\frac{r^{3}+29 r^{2}+243 r+559}{16(r+7)(r+11)}$ for any $r \in \mathbf{Q}-\{-7,-11\}$, the dynatomic polynomial $\Phi_{3}(z, c)$ is still irreducible in these cases. This demonstrates that $G_{N, c} \cong$ $G_{N}$ is not a necessary condition for $\left(\phi_{2, c}, 3, K / \mathbf{Q}\right)$ to satisfy the Galois-dynamics correspondence. In fact, we did not need to know anything about $\Sigma_{2,3}$ to prove Lemma 4.2

\subsection{Period 4.}

Lemma 4.5. The tuple $\left(\phi_{2, c}, 4, K / \mathbf{Q}\right)$ satisfies the Galois-dynamics correspondence for Galois number fields $K$ and $c \in \mathbf{Q}$ in the following cases:

(a) if $[K: \mathbf{Q}]=2$, or

(b) if $[K: \mathbf{Q}]>2$ and $c \notin\left\{\frac{-s^{3}-2 s+4}{4 s} \mid s \in \mathbf{Q}^{\times}\right\}$.

Proof. (a): There is an explicit complex parametrization of 4-cycles of $\phi_{2, c}$ due to Netto [Net00, Morton [Mor98a, and Erkama Erk06. Panraksa Pan11, Theorem 1.5.1.] used this parametrization to study the factorization of $\Phi_{4}(z, c)$ and prove that for a quadratic number field $K, c \in \mathbf{Q}$, and a 4-cycle $\left\{z_{0}, z_{1}, z_{2}, z_{3}\right\} \subset K$ of $\phi_{2, c}, z_{0}$ and $z_{2}$ are Galois conjugates. Therefore, $\left(\phi_{2, c}, 4, K / \mathbf{Q}, z_{0}\right)$ satisfies the Galois-dynamics correspondence. But $z_{0}$ is an arbitrary member of an arbitrary 4-cycle, so $\left(\phi_{2, c}, 4, K / \mathbf{Q}\right)$ satisfies the Galois-dynamics correspondence.

(b): Krumm [Kru18, Proposition 4.8] showed that

$$
\Sigma_{2,4}=\left\{-\frac{5}{2}\right\} \cup\left\{\frac{s^{2}+2 s-4}{8 s} \mid s \in \mathbf{Q}^{\times}\right\} \cup\left\{\frac{-s^{3}-3 s+4}{4 s} \mid s \in \mathbf{Q}^{\times}\right\},
$$

with explicit presentations of the exceptional Galois groups as subgroups of $S_{12}$. In fact, $\Phi_{4}(z, c)$ is still irreducible when $c \in\left\{-\frac{5}{2}\right\} \cup\left\{\frac{s^{2}+2 s-4}{8 s} \mid s \in \mathbf{Q}^{\times}\right\}$, even though it generates a different Galois group. By Proposition 1.5 $\left(\phi_{2, c}, 4, K / \mathbf{Q}\right)$ still satisfies the Galois-dynamics correspondence in those cases.

Remark 4.6. The factorization types of $\Phi_{4}(z, c)$ with $c \in \mathbf{Q}$ are explicitly known due to Morton [Mor98a, Theorem 4], Panraksa [Pan11, Theorem 2.3.5.], and Krumm Kru18, Theorem 1.2]. In particular, if $c \in\left\{\frac{-s^{3}-3 s+4}{4 s} \mid s \in \mathbf{Q}^{\times}\right\}$then $\Phi_{4}(z, c)$ factors either into an irreducible degree 8 polynomial and an irreducible degree 4 polynomial, or an irreducible degree 8 polynomial and two irreducible quadratic polynomials.

4.4. Period 5, 6, 7, and 9 .

Lemma 4.7. The tuple $\left(\phi_{2, c}, N, K / \mathbf{Q}\right)$ satisfies the Galois-dynamics correspondence for all nontrivial Galois number fields $K$ and for all but finitely many $c \in \mathbf{Q}$.

Proof. For $N \in\{5,6,7,9\}$, the exceptional set $\Sigma_{2, N}$ is finite due to Krumm Kru19. Since $\Phi_{N}(z, c)$ is necessarily irreducible for $c \notin \Sigma_{2, N}$, we are done by Proposition 1.5.

Remark 4.8. While an explicit description of $\Sigma_{2, N}$ is not yet known, Krumm Kru19, Section 9] provides an algorithm for computing the elements of $\Sigma_{2, N}$ of bounded naive height. 
Remark 4.9. For $d=2$ and $N \geq 5$, it is not known whether any class of pairs $\left(\phi_{2, c}, N\right)$ satisfies the Galois-dynamics correspondence besides those satisfying the irreducibility criteria and those explicitly found by computational searches for periodic points of exact period 5 and 6 [FPS97, HI13. Even when $K / \mathbf{Q}$ is required to be quadratic, there is an active field of research on understanding the structure of the dynamics of $\phi_{2, c}$ (cf. Doy18, Doy19, Doy20, DFK14, HI13, Kru16]).

\section{Acknowledgments}

The author would like to express thanks to Niccolò Ronchetti for introducing him to the field of arithmetic dynamics and to Zhiming Wang for the many preliminary conversations and projects that helped shape this project. The author is also grateful to Brian Conrad, Xander Faber, and John Doyle for numerous suggestions and clarifications. The author is also thankful to the anonymous referees for pointing out mistakes in earlier versions of this paper and for several insightful comments.

This work is based on a project that began under the support of the Office of the Vice Provost for Undergraduate Education of Stanford University and was later continued under the support of the National Science Foundation Graduate Research Fellowship Program under Grant No. DGE-1644869. Any opinions, findings, and conclusions or recommendations expressed in this material are those of the author and do not necessarily reflect views of the National Science Foundation.

\section{REFERENCES}

[BG17] Andrew Bridy and Derek Garton. Dynamically distinguishing polynomials. Res. Math. Sci., 4:Paper No. 13, 17, 2017.

[BL14] Xavier Buff and Tan Lei. The quadratic dynatomic curves are smooth and irreducible. In Frontiers in complex dynamics, volume 51 of Princeton Math. Ser., pages 49-72. Princeton Univ. Press, Princeton, NJ, 2014.

[Bou92] T. Bousch. Sur quelques problèmes de dynamique holomorphe. PhD thesis, Université de Paris-Sud, Centre d'Orsay, 1992.

[DFK14] John R. Doyle, Xander Faber, and David Krumm. Preperiodic points for quadratic polynomials over quadratic fields. New York J. Math., 20:507-605, 2014.

[Doy18] John R. Doyle. Preperiodic points for quadratic polynomials with small cycles over quadratic fields. Math. Z., 289(1-2):729-786, 2018.

[Doy19] John R. Doyle. Dynamical modular curves for quadratic polynomial maps. Trans. Amer. Math. Soc., 371(8):5655-5685, 2019.

[Doy20] John R. Doyle. Preperiodic points for quadratic polynomials over cyclotomic quadratic fields. Acta Arith., 196(3):219-268, 2020.

[Erk06] Timo Erkama. Periodic orbits of quadratic polynomials. Bulletin of the London Mathematical Society, 38:804-814, 2006.

[FPS97] E. V. Flynn, Bjorn Poonen, and Edward F. Schaefer. Cycles of quadratic polynomials and rational points on a genus-2 curve. Duke Math. J., 90(3):435-463, 1997.

[GO14] Yan Gao and YaFei Ou. The dynatomic periodic curves for polynomial $z \leftrightarrow z^{d}+c$ are smooth and irreducible. Science China Mathematics, 57(6):1175-1192, 2014.

[HI13] Benjamin Hutz and Patrick Ingram. On Poonen's conjecture concerning rational preperiodic points of quadratic maps. Rocky Mountain J. Math., 43(1):193-204, 2013.

[Hin88] Marc Hindry. Autour d'une conjecture de Serge Lang. Invent. Math., 94(3):575-603, 1988.

[Kru16] David Krumm. A local-global principle in the dynamics of quadratic polynomials. International Journal of Number Theory, 12(8):2265-2297, 2016.

[Kru18] David Krumm. Galois groups in a family of dynatomic polynomials. Journal of Number Theory, 187:469 - 511, 2018.

[Kru19] David Krumm. A finiteness theorem for specializations of dynatomic polynomials. Algebra Number Theory, 13(4):963-993, 2019. 
[KS21] David Krumm and Nicole Sutherland. Galois groups over rational function fields and explicit Hilbert irreducibility. J. Symbolic Comput., 103:108-126, 2021.

[Lan65] Serge Lang. Division points on curves. Ann. Mat. Pura Appl. (4), 70:229-234, 1965.

[LS94] Eike Lau and Dierk Schleicher. Internal addresses in the Mandelbrot set and Galois groups of polynomials. Preprint, Institute for Mathematical Sciences, Stony Brook, \#19, 1994.

[McQ95] Michael McQuillan. Division points on semi-abelian varieties. Invent. Math., 120(1):143159, 1995.

[Mor92] Patrick Morton. Arithmetic properties of periodic points of quadratic maps. Acta Arithmetica, 62(4):343-372, 1992.

[Mor96] Patrick Morton. On certain algebraic curves related to polynomial maps. Compos. Math., 103(3):319-350, 1996.

[Mor98a] Patrick Morton. Arithmetic properties of periodic points of quadratic maps. II. Acta Arith., 87(2):89-102, 1998.

[Mor98b] Patrick Morton. Galois groups of periodic points. Journal of Algebra, 201(2):401 - 428, 1998.

[MP94] Patrick Morton and Pratiksha Patel. The Galois Theory of Periodic Points of Polynomial Maps. Proceedings of the London Mathematical Society, 68(2):225-263, 1994.

[Net00] Eugen Netto. Vorlesungen über Algebra II. Teubner, Leipzig, 1900.

[Pan11] Chatchawan Panraksa. Arithmetic dynamics of quadratic polynomials and dynamical units. PhD thesis, University of Maryland, College Park, 2011.

[Poo98] Bjorn Poonen. The classification of rational preperiodic points of quadratic polynomials over Q: a refined conjecture. Math. Z., 228(1):11-29, 1998.

[Poo99] Bjorn Poonen. Mordell-Lang plus Bogomolov. Invent. Math., 137(2):413-425, 1999.

[Ser00] Jean-Pierre Serre. Euvres Collected papers, volume IV, chapter Résumé des cours de 1985-1986, pages 33-38. Springer-Verlag, Berlin, 2000. 1985-1998.

[Ser08] J.P. Serre. Topics in Galois theory. Research Notes in Mathematics. A K Peters, 2008.

[Sto08] Michael Stoll. Rational 6-cycles under iteration of quadratic polynomials. LMS J. Comput. Math., 11:367-380, 2008.

[VH92] F Vivaldi and S Hatjispyros. Galois theory of periodic orbits of rational maps. Nonlinearity, 5(4):961, 1992.

[WR94] Ralph Walde and Paula Russo. Rational periodic points of the quadratic function $Q_{c}(x)=$ $x^{2}+$ c. Amer. Math. Monthly, 101(4):318-331, 1994.

[WZ15] Zhiming Wang and Robin Zhang. On quadratic periodic points of quadratic polynomials. ArXiv e-prints, page arXiv:1504.00985, 2015. Computational programs: https://github.com/surim14/arith-dyn

(Robin Zhang) Department of Mathematics, Columbia University

Email address: rzhang@math.columbia.edu 